\title{
A Research on Bus Load Forecasting with Multi-level Coordination Optimization and Weight Colligation Mechanism
}

\author{
Fuyao DENG ${ }^{1}$, Changning $\mathrm{SHAO}^{2}$, a , Lei WU${ }^{1}$, Ming $\mathrm{LI}^{1}$, Xing $\mathrm{LI}^{1}$ \\ ${ }^{1}$ Sichuan Electric Power Corporation, Chengdu, Sichuan 610041, China \\ ${ }^{2}$ Beijing Tsingsoft Innovation Technology Co., Ltd, Beijing 100085, China \\ aEmail: cnshao@tsingsoft.com.cn
}

\begin{abstract}
Keywords: Bus Load; Multi-level Forecasting; Coordination Optimization; OLS
\end{abstract}
\begin{abstract}
Multi-level forecasting of power load is an inevitable demand based on current hierarchical management of our power system. Multi-level forecasting of bus load particularly provides power flow calculation and off-line security check with data support. Aiming at declining the offset of bus load Multi-level forecasting, this paper established a two-way coordination optimization process based on Genetic Algorithm, raised two methods to optimize and adjust the coordination quantity and obtain high confidence level coordination forecasting results. Finally the effect of the process was tested and verified by practical examples.
\end{abstract}

\section{Introduction}

Precise bus load forecasting provides fundamental boundary condition for making day-ahead generation schedule, operating power flow calculation and off-line security check which is an essential method to promote refined management for the power system. The most significant features of bus load forecasting are less base and mass quantity, complex load component, sensitivity with outer factors and so on. These features lead much difficulty to bus load forecasting. Customarily single ordinary forecasting method cannot work well, however more reliable result can be achieved with coordination optimization on multi-level bus load forecasting result.

Based on current hierarchical management of our power system multi-level load forecasting coordination refers to multiple dimensions load forecasting for the whole power system or just some nodes in it, therefore the confidence level of forecasting result will be enhanced by coordination and colligation. One typical application of multi-level load forecasting is through two levels: First make a direct forecasting of the total grid, then accumulate each sub grid forecasting, and both total and sub grid forecasting can be well improved by reducing the unbalance between the two level prediction. While as the terminal of power flow, buses in the power system have more stringent and complex connections, so that the idea of system load coordination can be learned to optimize multi-level bus load forecasting.

Scholars have done much research on coordination forecasting. Paper [3] raises basic model and constraint of coordination optimization, and sums its substance up as linear programming problem; Paper [4] extends the single problem as multi-level and multi-dimension related coordination model, and raises single and multiple evaluation indexes. This paper is mainly aimed at multi-level bus load forecasting coordination, using Ordinary Least Square (OLS) to solve the coordination problem and colligate the forecasting results according to average load of multi-level bus, therefore more accurate and reliable forecasting will be achieved.

\section{Two-way Coordination Model}

First we build two-way coordination optimization mold towards adjacent buses which have direct physical connection, then we use OLS to solve the bus adjustment coefficient of bus load forecasting on every level. 


\section{Basic two-way coordination optimization model}

Assume the higher level bus load is $P_{0}$, the lower level n buses (which are directly connected to the higher level bus) load are $P_{1}, P_{2}, \cdots, P_{n}$. According to physical features of power grid, the high level bus load equals to the sum of lower bus load and line loss:

$$
P_{0}=\sum_{i=1}^{n} P_{i}+\Delta r
$$

In the equation, $\Delta r$ refers to line loss.

For forecasting, equation (1) is not generally available, Since the forecasting error always exists, there always exists an unbalance $\Delta Q$ between $P_{0}$ and $\sum_{i=1}^{n} P_{i}+\Delta r$. So equation (1) can be change into:

$$
\widehat{P}_{0}=\sum_{i=1}^{n} \widehat{P}_{i}+\Delta r+\Delta \widehat{Q}
$$

Two-way coordination can be described as that through $\hat{P}_{0}$ and $\hat{P}_{i}(i=1,2, \cdots, n)$ coefficient adjustment, the sum of squares of unbalance $\Delta \hat{Q}$ can be minimized and so that both sides of equation (1) can be adjacent. So the coordination optimization objective function can be described as below:

$$
\min f=\sum_{t=1}^{m}\left[k_{0} \widehat{P}_{0, t}-\left(\Delta r_{t}+\sum_{i=1}^{n} k_{i} \widehat{P}_{i, t}\right)\right]^{2}
$$

In the equation, $\widehat{P}_{0, t}, \widehat{P}_{i, t}$ refer to high and low level bus load forecasting at $t$ moment, $\Delta r_{t}$ refers to line loss at moment, $k_{i}(i=0,1,2, \cdots, n)$ refers to adjustment coefficient of original forecasting, $\mathrm{m}$ refers to sample point number in one day.

$$
\min f=\sum_{t=1}^{m}\left[k_{0} \widehat{P}_{0, t}-\left(\Delta r_{t}+\sum_{i=1}^{n} k_{i} \widehat{P}_{i, t}\right)\right]^{2}
$$

\section{Linear OLS Solution}

The nature of basic model of adjacent bus load forecasting coordination as equation (3) describes is optimization. There are different solutions against different optimization characteristics such as Analytic method based on OLS, iterative method based on Gradient Descent(GD),and heuristic algorithm for complex constraint and local optimum problems like simulated annealing and genetic algorithm. This case is a typical Linear Programming (LP) problem that can be solved directly by OLS. According to extreme value theory of multivariate function, objective equation (3) satisfies the equation as below:

$$
\left\{\begin{array}{c}
\frac{\partial f}{\partial k_{0}}=2 \sum_{t=1}^{m} \widehat{P}_{0, t}\left[k_{0} \widehat{P}_{0, t}-\left(\Delta r_{t}+\sum_{i=1}^{n} k_{i} \widehat{P}_{i, t}\right)\right]=0 \\
\frac{\partial f}{\partial k_{1}}=-2 \sum_{t=1}^{m} \widehat{P}_{1, t}\left[k_{0} \widehat{P}_{0, t}-\left(\Delta r_{t}+\sum_{i=1}^{n} k_{i} \widehat{P}_{1, t}\right)\right]=0 \\
\vdots \\
\frac{\partial f}{\partial k_{n}}=-2 \sum_{t=1}^{m} \widehat{P}_{n, t}\left[k_{0} \widehat{P}_{0, t}-\left(\Delta r_{t}+\sum_{i=1}^{n} k_{i} \widehat{P}_{n, t}\right)\right]=0
\end{array}\right.
$$

set: 


$$
\boldsymbol{P}=\left(\begin{array}{cccc}
\sum_{t=1}^{m} \hat{P}_{0, t} & -\sum_{t=1}^{m} \hat{P}_{1, t} & \cdots & -\sum_{t=1}^{m} \widehat{P}_{n, t} \\
-\sum_{t=1}^{m} \hat{P}_{0, t} & \sum_{t=1}^{m} \hat{P}_{1, t} & \cdots & \sum_{t=1}^{m} \hat{P}_{n, t} \\
\vdots & \vdots & \ddots & \vdots \\
-\sum_{t=1}^{m} \hat{P}_{0, t} & \sum_{t=1}^{m} \hat{P}_{1, t} & \cdots & \sum_{t=1}^{m} \hat{P}_{n, t}
\end{array}\right)
$$

set adjustment coefficient vector : $\boldsymbol{k}=\left(k_{0}, k_{1}, \cdots k_{n}\right)^{T}$,

line loss vector: $\boldsymbol{r}=\underbrace{\left(\sum_{t=1}^{m} r_{t}, \sum_{t=1}^{m} r_{t}, \cdots, \sum_{t=1}^{m} r_{t}\right)^{T}}_{n+1 \uparrow}$

so equation (4) can be transformed into:

$$
\boldsymbol{P} \boldsymbol{k}=\boldsymbol{r}
$$

the OLS result can be:

$$
\boldsymbol{k}=\boldsymbol{P}^{-1} \boldsymbol{r}
$$

\section{Multi-level bus weight colligation}

For $220 \mathrm{kV}, 110 \mathrm{kV}$ and other middle level bus, the adjacent bus load forecasting can be coordinated up and down for twice, and the two coordination results can be colligated to enhance the reliability of the forecasting.

High level bus usually has relatively large capacity, more precise load forecasting and less coordination, so its adjustment coefficient weight is small; Low level bus is on the contrary. So bus capacity can be used as the foundation of weight allocation. Considering a three-level bus structure, the capacities of high and low bus are $S_{\text {upper }}$ and $S_{\text {lower }}$, (quantity of low level buses is n), and the corresponding middle level bus upward and downward adjustment coefficients are $k_{\text {upper }}$ and $k_{\text {lower }}$.

So its adjustment coefficient weight colligation is:

$$
k=k_{\text {upper }} \cdot \frac{n S_{\text {lower }}}{S_{\text {upper }}+n S_{\text {lower }}}+k_{\text {lower }} \cdot \frac{S_{\text {upper }}}{S_{\text {upper }}+n S_{\text {lower }}}
$$

\section{Example analysis}

Use a certain bus of $220 \mathrm{kV}$ main transformer high-voltage side (Capacity $180 \mathrm{MVA}$ ) which is attached with $500 \mathrm{kV}$ bus (Capacity $1200 \mathrm{MVA}$ ) and $110 \mathrm{kV}$ bus (Capacity 50MVA) as an example for analysis. The real and forecasting bus load shows as Fig.2. (load has been normalized, the same as the following content) 


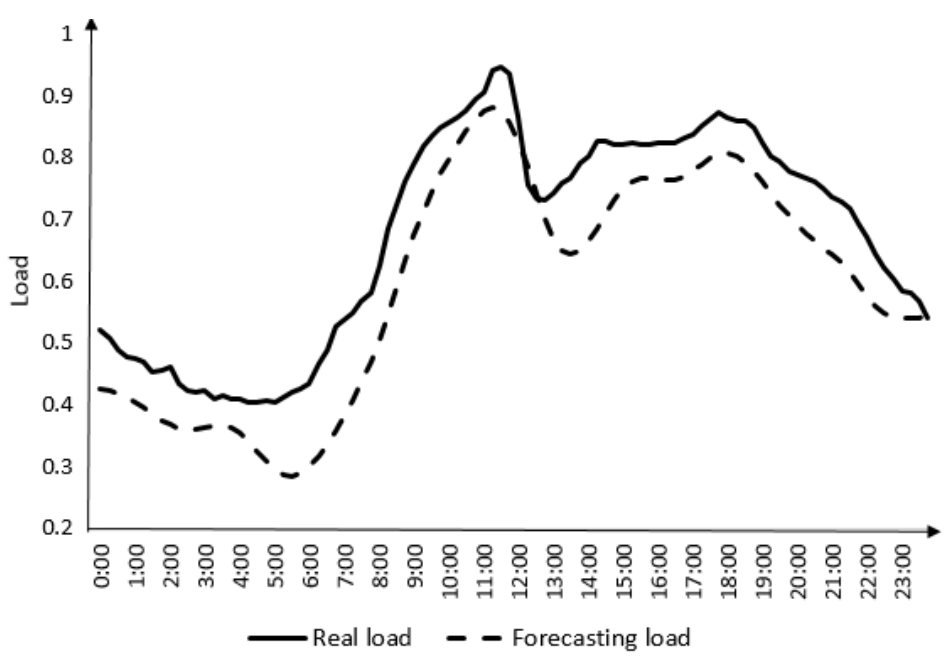

Fig.1. Real load and forecasting load curve

As Fig. 1 shows, forecasting load without coordination is collectively lower than the real load and certain period has rather larger deviation. Lines loss comes from statistics of historical real load, $\Delta r_{t}=1, t=1,2, \cdots, N$.

Make the two-way coordination of the $220 \mathrm{kV}$ bus load forecasting with $500 \mathrm{kV}$ upward and $110 \mathrm{kV}$ downward bus load forecasting according to the coordination model described in Section 1.Forecasting curve before and after coordination and the real load curve show as Fig.2.

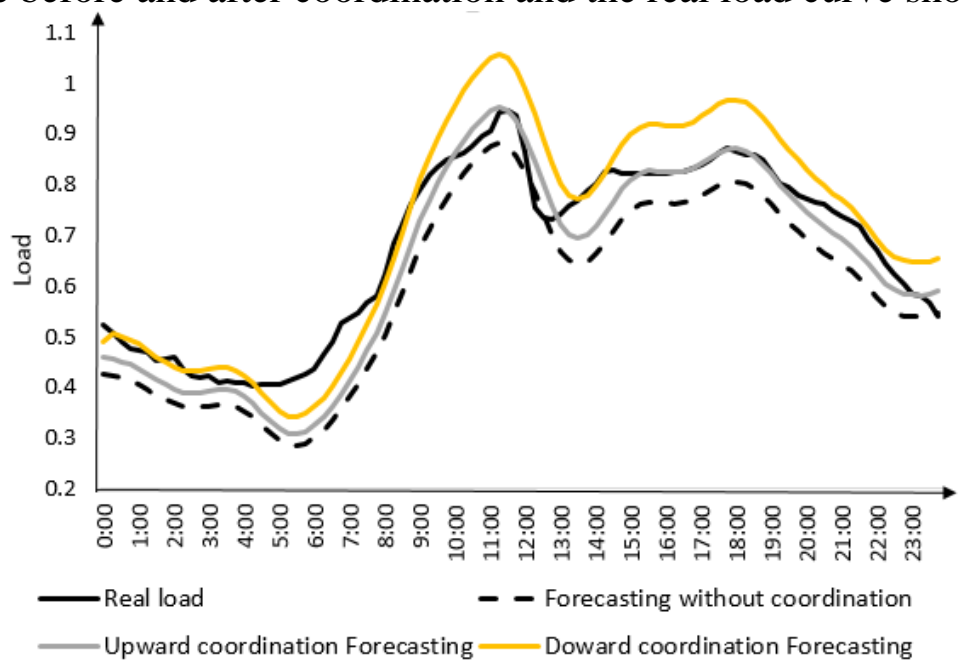

Fig.2. Upward and downward coordination effect

As Fig.2. shows, the coordination forecast result apparently performs better than the forecast result without coordination, and downward coordination effect is better than upward coordination. According to equation (6), the upward and the downward can be calculated:

$$
k_{\text {upper }}=1.15, \quad k_{\text {lower }}=1.08
$$

And according to equation (7), the weight colligation adjustment coefficient can be calculated: $k=1.096$

Weight coordination effect and forecasting accuracy comparison shows in Fig.4. 


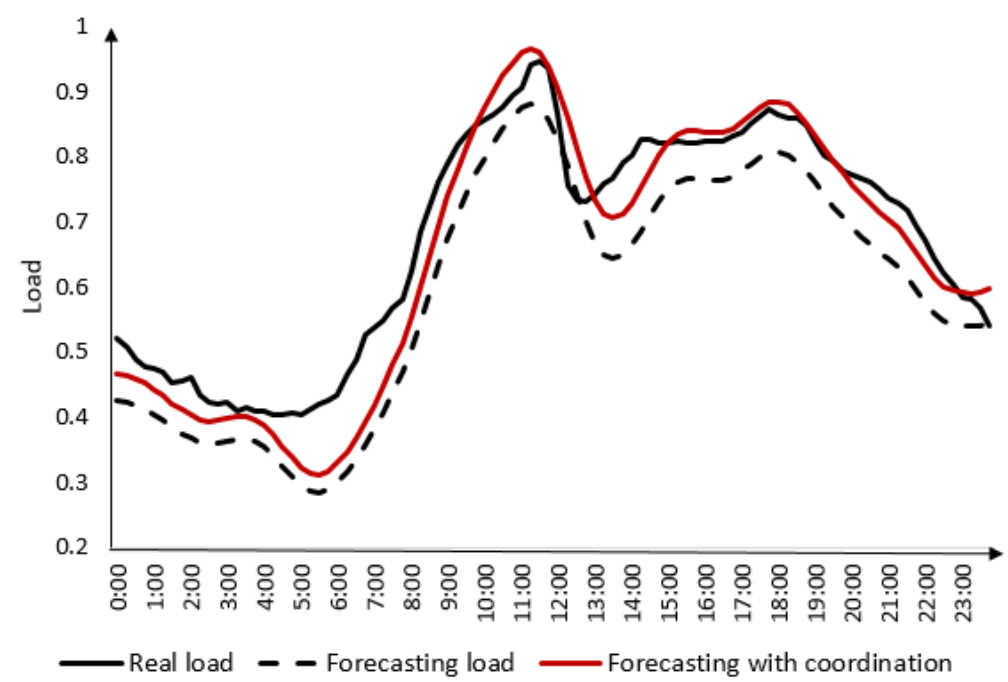

Fig.3. Weight coordination effect

Coordination effect and forecasting accuracy also can be showed in Tab.1.

Tab.1. Coordination coefficient and accuracy

\begin{tabular}{ccc}
\hline Coordination Status & Coordination coefficient & Accuracy (\%) \\
\hline No Coordination & - & $91.1 \%$ \\
Upward Coordination & 1.15 & $92.8 \%$ \\
Downward Coordination & 1.08 & $94.4 \%$ \\
Colligation Coordination & 1.096 & $94.8 \%$
\end{tabular}

As Tab.1. shows, the forecasting accuracy has large progress though the two-way coordination optimization raised by this paper, Moreover its application in multi-level bus load coordination colligation performs even better which is 3.7\% higher than the original forecasting.

\section{Conclusion}

This paper mainly studies multi-level bus load forecasting. Though the attempt of two-way bus load coordination which is physically attached, the coordination optimization weight colligation model based on bus capacity is established. Tested by the real example, this model proves that it can apparently improve the bus load forecasting, which will bring about significant progress of energysaving power generation dispatching and power flow optimization.

It should be pointed that in general the coordination model this paper raised is an overall coordination which performs weakly in detailed process. The point-to-point colligation refining coordination can help to solve the problem. Secondly though the study object of this paper is only a small occasion of bus load, the thinking can be promoted, including the coordination of upper and lower power grid and even multi-level grid which highly optimizes the load forecasting. These works need further study in the future.

\section{Reference}

[1] YANG Licai, ZHANG Wenlei, ZHOU Yong, LIAO Feng, XU Chengzhuo, CHENG Yiming, YAO Jiangang. Application of Indirect Prediction Method in Bus Load Forecasting, Power System Technology[J], 2011, 12:177-182.

[2] KANG Chongqing, ZHAO Ran, CHEN Xinyu, YANG Xingyu, CAO Xin, LIU Mei, Basic Analysis of Multi-level Load Forecasting[J], Power System Protection and Control, 2009,09:1-7.

[3] MOU Tao, KANG Chongqing, XIA Qing, John N. JIANG, LIU Mei. Multilevel Load 
Forecasting of Power System and Its Coordination Problem (II) Basic Coordination Model[J], Power System Automation, 2008,08:14-18.

[4] MOU Tao, KANG Chongqing, XIA Qing. Multilevel Load Forecasting of Power System and Its Coordination Problem (IV) Constrained Least Squares Confidence [J], Power System Automation, 2010, 02:43-47.

[5] FENG Li, QIU Jiaju. Festival Power Load Forecasting Based on Genetic Algorithm of Vague Muli-targets[J]. Journal of Chinese Electrical Engineering Science, 2005, 10:29-34.

[6] TANG Kezong, Improvement and Application of Genetic Algorithm and Particle Swarm Optimization Algorithm[D], Nanjing University of Science and Technology, 2011

[7] Amjady N. Short-term bus load forecasting of power systems by a new hybrid method[J]. Power Systems, IEEE Transactions on, 2007, 22(1): 333-341.

[8] Kassaei H R, Keyhani A, Woung T, et al. A hybrid fuzzy, neural network bus load modeling and predication[J]. Power Systems, IEEE Transactions on, 1999, 14(2): 718-724.

[9] Ashok S, Banerjee R. An optimization mode for industrial load management[J]. Power Systems, IEEE Transactions on, 2001, 16(4):

[10]XUE Jiliang, GU Jie, DU Jian, CHU Linlin, ZHANG Yujun, LU Huifeng. Multi-level Load Forecasting of Region Power Grid Based on Industrial Load Curve[J]. Journal of Electric Power System and Its Automation, 2014, 01:18-21 
